\title{
THE USES AND IRRELEVANCE OF HIGHER TAXA
}

DAMUTH, John, Dept. of Biological Sciences, University of California, Santa Barbara, CA 93106 U.S.A.

Different data and ways of ordering nature are relevant to different questions, and thus there is no single classification of natural phenomena appropriate for all questions in macroevolution.

Consider two distinct, coexisting modes of causal explanation in paleontology. The historical mode takes the form of a narrative, a sequence of events or situations, each one "explaining" the following event as a natural or obvious outcome. Traditionally, the historian of human society usually seeks explanations in terms of transitions from one state to the next. It is thus natural for the paleontologisthistorian to regard the primary problems of macroevolution as involving the transformation of character states and the sequence of branching lineages. The data summarized by a cladogram are thus of direct relevance to a vast number of macroevolutionary questions requiring historical-mode answers. However, taxa per se, as groupings of organisms based on specific criteria, are not sequences of events and thus are not particularly relevant to these kinds of questions. In contrast, a functional (or mechanistic, or process) mode of explanation characterizes most of the rest of natural science. One may define a mechanism as a specified set of causal interactions among entities, controlled by parameters. The essence of a mechanism is that given the parameter values, the result is inevitable and predictable, and the process continues to "completion." The entities that are functioning parts within the mechanism, and among which causal relations are specified, must usually satisfy the criteria of historical contemporaneity, geographic propinquity, and capacity to interact in the ways specified. There is no requirement involving common descent. The paleontologist seeking answers to questions about mechanism must therefore be interested primarily in functional (usually ecological) entities (organisms, populations, communities, biotas) and their environments rather than taxa per se or extended sequences of splitting events. Historical sequences involving ecological entities may often be more informative about ecologically based processes than sequences reflecting character transformations within lineages, because an ecological entity, by definition, occurs in a specific environment that may be shared by other ecologically interacting entities.

Higher taxa thus have little relevance to explanation in macroevolution, although scientifically useful groupings of species may sometimes correspond to taxa defined with regard to common descent or other criteria. Pre-Darwinian taxa were groupings of species thought to reflect ideal (usually Divine) ordering principles. The idea that the nested monophyletic groups extracted from a cladogram form the only "real" scientific groupings of species is a vestige of this pre-Darwinian idealism. This metaphysical issue is irrelevant. Entities have meaning, or not, only with regard to particular scientific questions or mechanisms. Paraphyletic taxa have no meaning in cladistic practice. Likewise, it is difficult, if not impossible, to define the general functional role of clades, or to give a general biological interpretation of their fates. The value of a cladogram is not that it defines monophyletic taxa (which seem largely irrelevant to macroevolution), but that it represents a hypothesized sequence of historical events (which is relevant).

Traditional, "synthetic" taxa that may be paraphyletic are, like all taxa based on common descent, usually inappropriate candidates for interactors in macroevolutionary mechanisms. An exception may be the way synthetic taxa are analyzed statistically in large-scale taxonomic diversity studies, where they can stand in for more appropriate species-level or populational data that are not readily available. Studying extinction of clades rather than synthetic taxa is not approaching the same question with "better" data, but constitutes asking a different question - and one whose answer may be even less easily causally interpretable. Before expunging synthetic taxa from our collective memory, we should use them to gain whatever perspective they can give on the causes of major events in life's history. 